\title{
May fermented Baltic Sea herring help in conditions of gut disorders, such as gastric catarrh and heartburn?
}

\author{
Jan Olof Gustav Karlsson ${ }^{1}$, Jan Eriksson ${ }^{2}$ \\ ${ }^{1}$ Division of Drug Research/Pharmacology, Linköping University, Linköping, Sweden \\ ${ }^{2}$ Hornberje Holding AS, Österfärnebo, Sweden
}

\begin{abstract}
Background: It has been suggested that disruption of the gut microbiota can be significant with respect to pathological intestinal conditions, such as irritable bowel syndrome (IBS), gastric catarrh (GC), and heartburn (HB). Through history, an essential part of the colonization of the human gut took place by ingestion of food preserved by fermentation. The natural replenishment of microbes via food and beverage is today low because food is "sterilized" through boiling, broiling, and pasteurization. Modulating the gut microbiota with fermented food products may hence be considered as a strategy to treat such conditions. Fermented Baltic Sea herring (FBSH) is an example of a Lactobacillusfermented food product, which was tested in the present study.

Methods: A 30-day open study was performed in 42 volunteers with IBS, GC, or HB. Volunteers were recruited by advertisements in daily newspapers. The volunteers were provided with gelatin capsules for the study, each containing approximately $100 \mathrm{mg}$ freeze dried FBSH. They were also provided with forms that contained columns and rows for every test day where the volunteers were ask to fill in number of capsules taken, and to report possible improvements according to a $0-10$ scale, where 10 stands for full recovery.

Results: The most reported common disorder symptom was IBS and 7 of 14 of these volunteers reported recovery, with a mean recovery of 4.4. All of the 9 volunteers reported recovery from GC, with a mean recovery of 8.4. Five of 6 volunteers reported recovery from $\mathrm{HB}$, with a mean recovery of 6.8 .

Conclusion: Although the present study is a small open study, the overall results are exciting and merits further studies in volunteers, ideally in a double-blind placebo-controlled manner.
\end{abstract}

\section{ARTICLE HISTORY}

Received 01 May 2018

Accepted 15 June 2018

Published 21 June 2018

\section{KEYWORDS}

Butyric acid; fermented Baltic Sea herring; gastric catarrh; gut microbiota; heartburn; irritable bowel syndrome; lactobacillus fermented; open study; probiotics; volunteers

\section{Introduction}

Controlled clinical trials have identified probiotics that favorably prevent or improve the symptoms of various gut disorders including inflammatory bowel disease, irritable bowel syndrome (IBS), and infectious and antibiotic-associated diarrhea [1]. Most of these products are supplemented milk products. The list of probiotic microorganisms is long and includes various species of Lactobacillus, Bifidobacterium, Streptococcus, Saccharomyces boulardii, and E. coli [1]. The used bacteria are mainly chosen because milk is an ideal medium for them to grow in and give tasty products. The World Health Organization defines probiotics as "live microorganisms which when administered in adequate amounts confer a health benefit on the host." However, diet per se is extremely important in shaping the human gut microbiota, one of the most densely populated microbial ecosystems in nature [2].

The natural replenishment of microbes via food and beverage is today low because food is "sterilized" through boiling, broiling, and pasteurization.

Contact Jan Olof Gustav Karlsson $₫$ janolof.karlsson@ktias.com Division of Drug Research/Pharmacology, Linköping University, Linköping, Sweden

(C) EJManager. This is an open access article licensed under the terms of the Creative Commons Attribution Non-Commercial License (http:// creativecommons.org/licenses/by-nc/3.0/) which permits unrestricted, noncommercial use, distribution and reproduction in any medium, provided the work is properly cited. 
Moreover, the technique to preserve food by fermentation is essentially forgotten among modern people.

Fermented Baltic Sea herring (FBSH) is an example of a Lactobacillus-fermented food product. The oldest archeological finding of fish fermentation is more than 9,000 years old [3]. FBSH or surströmming in Swedish (combination of sour and the local name of herring, strömming, in the Baltic Sea) seems to date back to at least the fourteenth century [4]. The fermentation is a result of interplay between the bacteria and gut enzymes from the Baltic Sea herring, according to the Swedish National Food Agency. The fermentation results in pungent smelling acids are formed in the fish such as propionic acid, butyric acid, and acetic acid [4]. Hydrogen sulfide is also produced. The osmotic pressure of the brine rises above the level where bacteria responsible for rotting can thrive and prevents decomposition of fish proteins. This condition enables Haloanaerobium bacteria to prosper and decompose the fish glycogen into organic acids, making it further acidic. The Swedish National Food Agency conducted trials in the 1970s by adding known food pathogens such as Staphylococcus aureus, Bacillus cereus, and Clostridium perfringens to FBSH, but none of these microorganisms could be shown to grow in FBSH, thus indicating that there is an efficient barrier towards growth of unwanted bacteria in FBSH [4]. For more details the reader is referred to [4].

There are to the best of our knowledge no scientific studies showing beneficial effects of FBSH. In fact, a search at PubMed using the search term "FBSH" results in 0 hits, whereas "probiotics" results in almost 15,000 hits. Nevertheless, one of us (JE, better known as Skogsjan) got the idea that FBSH may cure certain stomach problems, such as heartburn (HB) and gastritis, many years ago. JE observed that these problems seemed to improve after consumption of FBSH. JE hence initiated more systematic studies and found further support for his idea. However, JE also realized that treatment with FBSH due to its pungent smell was associated with invincible problems for most individuals to consume it. Except for people living in certain parts of Sweden, there are relatively few that consume FBSH. He hence developed a method enabling FBSH to be freeze dried and encapsulated $\left(\right.$ EpicAid $^{\circledR}$ ), as described in a US patent (US 6,572,883 B1). As an early part of the developmental process, the present study in volunteers was conducted in 2004. During that time the company Rebiotica $A B$ was formed and the production of EpicAid was scaled up and prepared for marketing. A manufacturing plant was established in Sandviken Municipality, Sweden. However, at that time the Baltic Sea herring contained higher levels of dioxins and polychlorinated biphenyls (PCBs) than the permitted levels for fish in the European Union (EU). Although Sweden was granted exceptions from these rules when it came to marketing of Baltic Sea herring as foodstuff, a local supervisory authority in Sandviken (Miljö och Hälsoskyddsnämnden) surprisingly decided to stop Rebiotica from marketing EpicAid, i.e., the freeze-dried form of FBSH. Rebiotica AB complained to the Swedish Responsible Authority, the National Food Agency, but the Agency maintained the local decision. This decision halted any further development of the product, and the results from the above-mentioned study were not published at that time. However, today selected Baltic Sea herring is available that contains less dioxins and PCBs, well within the EU's rules, and it is in this context results from the study is now published. JOGK came into the project soon after the in-life phase of the present study and drafted the raw data, which now has been compiled into an article.

\section{Materials and Methods}

An open 30-day study was performed during 2004 in volunteers with IBS, gastric catarrh (GC), HB, and other related but undefined stomach problems. Forty-two volunteers (22 females and 20 males) were recruited by advertisements in the daily newspapers "Gefle Dagblad" and "Dagens Industri." Prior to the start of the study, each volunteer filled out a form with her/his name, address, telephone number, birth date, disease symptoms, and possible use of medicines; only medicines relevant to stomach problems are given in the Results section. There is no information in the raw data whether or not the symptoms have been confirmed by a physician. Because symptoms from GC and HB are rather clear, lack of this information is probably of minor importance. On the other side as discussed later on, diagnosis of IBS is highly problematic even for physicians. The volunteers were provided with capsules for the study, each containing approximately $100 \mathrm{mg}$ freeze dried FBSH. FBSH, manufactured at Oscars Surströmming, Söråker, Sweden were used. The FBSH was divided into small pieces and freezedried by means of a conventional freeze-drying equipment. After freeze-drying, the herring was 
grinded, and subsequently, encapsulated in gelatine capsules, essentially impermeable to the scent emitted from the powder, as described in the US patent 6,572,883 B1. The volunteers were provided with forms that in addition to volunteers' ID (name plus birth date) contained columns and rows for every test day where the volunteers were ask to fill in the number of capsules taken in the morning and in the evening (they were recommended to take 1-6 capsules twice daily $(2 \times 1-6)$ based on the necessity), and to report possible improvements according to a 0-10 scale, where 0 stands for no change and 10 for complete recovery from the disease symptoms. Thirty-one volunteers (20 females and 11 males) fulfilled the study and returned the forms. Thirty of them were eligible for inclusion in the study and one was excluded because of protocol violation. Statistical difference where appropriate was tested by a two-tailed $t$-test.

\section{Results}

Thirty-one patients were recruited to participate in the study, 20 females (22-63 years old) and 11 males (31-64 years old) (Table 1). Some protocol violations occurred but these were relatively minor and mainly of the character "data missing" regarding medications. The most common disorder symptom (alone or in combination with other symptoms) was IBS. Seven of 14 volunteers with IBS reported recovery from the symptoms and the mean ( \pm S.E.M.) recovery was $4.4 \pm 1.1$, corresponding to $44 \%$. Nine of 9 volunteers reported recovery from GC (alone or in combination with other symptoms) with a mean \pm S.E.M. recovery of $8.4 \pm 0.6$ $(84 \%)$. Five of 6 volunteers reported recovery from HB (gastroesophageal reflux; alone or in combination with other symptoms) with a mean ( \pm S.E.M.) recovery of $6.8 \pm 1.5$ (68\%). The results are summarized in Figure 1. The difference between the IBS and the GC group was statistically significant ( $p=0.0124$; two-tailed $t$-test).

Six out of 9 non-responders had IBS symptoms the other four had HB, undefined stomach problems, and hiatus hernia, respectively. The underlying mechanisms behind IBS are heterogeneous and available treatment unsatisfactory [5]. Although changes in microbiota may play an important role in IBS many other factors seem to be of importance. This heterogeneity may explain the lower numbers of responders in the volunteers with IBS than in volunteers with GC and HB.

\section{Discussion}

To the best of our knowledge, this is the first study investigating the effects of FBSH on GC, HB, and IBS. Although the present feasibility study is an open non-placebo controlled study, the results are interesting. The beneficial effect on GC and HB is astonishing and we do not believe that this effect is a pure placebo effect. The significantly lower efficacy in the IBS group compared to the GC group may in fact serve as a "built-in" control, with respect to placebo effects.

Our relation to microorganisms is ancient, and the interplay between the microorganisms and the host was an important part of the evolution of multicellular eukaryotes. In terms of cell number, adult humans are more prokaryotic than eukaryotic with $90 \%$ of our cells estimated to be of microbial origin, and only $10 \%$ of human origin [6-9]. It has been estimated that our gut contains in the range of 1,000 bacterial species and 100 -fold more genes than are found in the human genome. This community is commonly referred to as our hidden metabolic "organ" due to its immense impact on human wellbeing, including host metabolism, physiology, nutrition, and immune function, and protection of the colonized host against invasion by alien microbes $[1,9,10]$. It is now generally accepted that the "central genome dogma," i.e., a causal chain going from DNA to RNA to proteins and downstream to biological functions, should be replaced by the "fluid genome dogma," that is, complex feed-forward and feed-back cycles that interconnect organism and environment by epigenomic programing and reprograming throughout life and at all levels [8]. The epigenomic programing is the net sum of interactions derived from our own metabolism and microbiota as well as external factors such as diet, pharmaceuticals, environmental compounds, and so on. Foods and gut microbiota are the two most important environmental factors in epigenomic programing, and are most pronounced in pregnancy and early in life.

Colonization of the gut starts already during normal delivery, where the infant is exposed for large amounts of maternal microbes [1,9,11]. During the following colonization and up to an age of 2 years, more than 1,000 species will be established in the gut. Through history, an essential part of the colonization of the human gut took place by ingestion of food preserved by fermentation. There are good reasons to anticipate that food earlier contained 
Table 1. Baseline characteristics, FBSH daily dose (number of capsules), and outcome (recovery) in each volunteer.

\begin{tabular}{|c|c|c|c|c|c|c|}
\hline Volunteer & $\begin{array}{l}\text { Gender } \\
\text { (F/M) }\end{array}$ & $\begin{array}{c}\text { Age } \\
\text { (years) }\end{array}$ & Symptoms & Medications & $\begin{array}{l}\text { Daily dose } \\
\text { (capsules) }\end{array}$ & $\begin{array}{c}\text { Recovery }^{\mathrm{a}} \\
(0-10)\end{array}$ \\
\hline$\overline{\# 1}$ & $M$ & 49 & $\mathrm{HB}^{\mathrm{b}}$ & No medications & $2 \times 2$ & 10 \\
\hline$\# 2$ & $\mathrm{~F}$ & 22 & $\mathrm{GC}^{\mathrm{c}}$ & Data missing & $2 \times 2$ & 8 \\
\hline \#3 & $\mathrm{F}$ & 39 & $\mathrm{IBS}^{\mathrm{d}}$ & No medications & $2 \times 4$ & 0 \\
\hline$\# 4^{\mathrm{e}}$ & $\mathrm{F}$ & 63 & $\mathrm{HB}$ & Omeprazol $^{f}$ & $2 \times 2$ & 0 \\
\hline$\# 5$ & M & 31 & $\mathrm{HB}+$ Anxious stomach & No medications & $2 \times 2$ & 6 \\
\hline$\# 6$ & $\mathrm{~F}$ & 50 & IBS & No medication & $\begin{array}{c}2 \times 2(\text { w. 1) } \\
2 \times 4(\text { w. } 2-4)\end{array}$ & 7 \\
\hline \#7 & M & 64 & GC & Ranitidine $^{\mathrm{g}}$ & $2 \times 4$ & 7 \\
\hline$\# 8^{h}$ & $\mathrm{~F}$ & 47 & $\mathrm{HB}+\mathrm{GC}$ & Lansoprazol $^{f}$ & $2 \times 4$ & 10 \\
\hline$\# 9$ & $\mathrm{~F}$ & 24 & IBS & No medications & $2 \times 4$ & 0 \\
\hline$\# 10^{i}$ & $\mathrm{~F}$ & 53 & IBS & No medications & $\begin{array}{c}2 \times 3(\text { w. } 1-2) \\
2 \times 4(\text { w. } 3)\end{array}$ & 0 \\
\hline$\# 11^{\mathrm{h}}$ & $\mathrm{F}$ & 49 & IBS & No medications & $2 \times 3$ & 6 \\
\hline$\# 12$ & $\mathrm{~F}$ & 60 & Undefined stomach pr. & Data missing & $2 \times 2-3$ & 10 \\
\hline$\# 13$ & $\mathrm{~F}$ & 58 & $\mathrm{IBS}+\mathrm{HB}$ & No medications & $2 \times 2$ & 7 \\
\hline$\# 14$ & $\mathrm{~F}$ & 35 & $\mathrm{IBS}+\mathrm{HB}+\mathrm{GC}$ & $\begin{array}{l}\text { No medications (prev. } \\
\text { omeprazolf }{ }^{f} \text { ) }\end{array}$ & $\begin{array}{c}2 \times 2(\text { w. 1) } \\
2 \times 4(\text { w. } 2-4)\end{array}$ & 8 \\
\hline$\# 15$ & $\mathrm{~F}$ & 54 & $\mathrm{GC}+$ ulcerous colitis & $\begin{array}{l}\text { Combizym (digestion } \\
\text { enzymes mixture) }\end{array}$ & $2 \times 2$ & 10 \\
\hline$\# 16^{\mathrm{e}}$ & M & 50 & IBS & $\begin{array}{l}\text { No medication } \\
\text { (normally ranitidines) }\end{array}$ & $2 \times 2$ & 0 \\
\hline$\# 17$ & $\mathrm{~F}$ & 39 & IBS & No medications & $2 \times 2$ & 7 \\
\hline$\# 18$ & M & 59 & GC & Esomeprazol $^{f}$ & $2 \times 2(w .1)$ & \\
\hline & & & & & $\begin{array}{c}2 \times 3(\text { w. 1) } \\
2 \times 4(\text { w. } 2-4)\end{array}$ & 5 \\
\hline$\# 19^{j}$ & M & 32 & GC & $\begin{array}{l}\text { No medications (prev. } \\
\text { omeprazolf }^{f} \text { ) }\end{array}$ & $\begin{array}{c}2 \times 2(\text { w. 1) } \\
2 \times 4(\text { w. } 1-4)\end{array}$ & 9 \\
\hline$\# 20$ & $\mathrm{~F}$ & 31 & IBS & No medications & $\begin{array}{l}2 \times 6(\text { w. 1-2) } \\
2 \times 3(\text { w. 3-4) }\end{array}$ & 10 \\
\hline$\# 21$ & M & 43 & GC + peptic ulcer disease & $\begin{array}{l}\text { Lansoprazolf } \text { (prev } \\
\text { ranitidine }^{\mathrm{g}} \text { ) }\end{array}$ & $2 \times 4$ & 9 \\
\hline$\# 22$ & $\mathrm{~F}$ & 49 & GC & No medications & $\begin{array}{c}2 \times 2(\text { w. } 1) \\
2 \times 1(\text { w. } 2-4)\end{array}$ & 10 \\
\hline$\# 23$ & $\mathrm{~F}$ & 52 & IBS & Dimetikon and Lact Bac & $\begin{array}{l}2 \times 2 \text { (w. 1) } \\
2+3 \text { (w. 2) } \\
2 \times 3 \text { (w. 3) } \\
2 \times 4 \text { (w. 4) }\end{array}$ & 8 \\
\hline$\# 24$ & $\mathrm{~F}$ & 23 & IBS & No medications & $\begin{array}{c}2 \times 1(\text { w. } 1) \\
2 \times 2(\text { w. } 2-4)\end{array}$ & 0 \\
\hline$\# 25$ & $\mathrm{~F}$ & 46 & IBS & No medications & $\begin{array}{c}2 \times 1 \text { (w. 1) } \\
2 \times 2(\text { w. } 2-3) \\
2 \times 3(\text { w. } 4)\end{array}$ & 0 \\
\hline$\# 26^{k}$ & M & 61 & IBS & No medications & $2 \times 2$ & - \\
\hline$\# 27$ & $\mathrm{~F}$ & 46 & Undefined stomach pr. & Data missing & $2 \times 2-4$ & 0 \\
\hline$\# 28$ & M & 47 & IBS & No medications & $2 \times 1$ & 9 \\
\hline$\# 29$ & M & 47 & Hiatus hernia' & $\begin{array}{l}\text { Lansoprazol }^{f} \\
\text { (prev. omeprazol) }\end{array}$ & $\begin{array}{l}2 \times 2 \\
2 \times 4\end{array}$ & 0 \\
\hline \#30 & M & 35 & Undefined stomach pr. & Data missing & Data missing & 6 \\
\hline$\# 31$ & $\mathrm{~F}$ & 31 & Undefined stomach pr. & Data missing & $2 \times 1$ & 7 \\
\hline
\end{tabular}

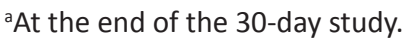

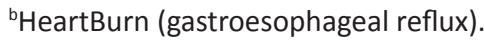

'Gastric Catarrh (gastritis).

'Irritable Bowel Syndrome.

eInterrupted the study after 2 weeks.

${ }^{\mathrm{f}} \mathrm{H}^{+} / \mathrm{K}^{+}$-ATPase proton pump inhibitor.

$\mathrm{g}_{2}$-receptor antagonist.

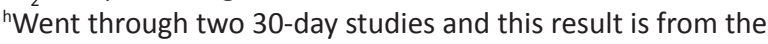
second study.
IInterrupted the study after 3 weeks.

interrupted in the middle of week 3; the given recovery (9) is from that time point.

"Filled in the scheme incorrect with respect to recovery; used " $\mathrm{X}$ " instead of a figure between $0-10$.

'Protrusion of a part of the stomach through the diaphragm at the esophageal opening. 


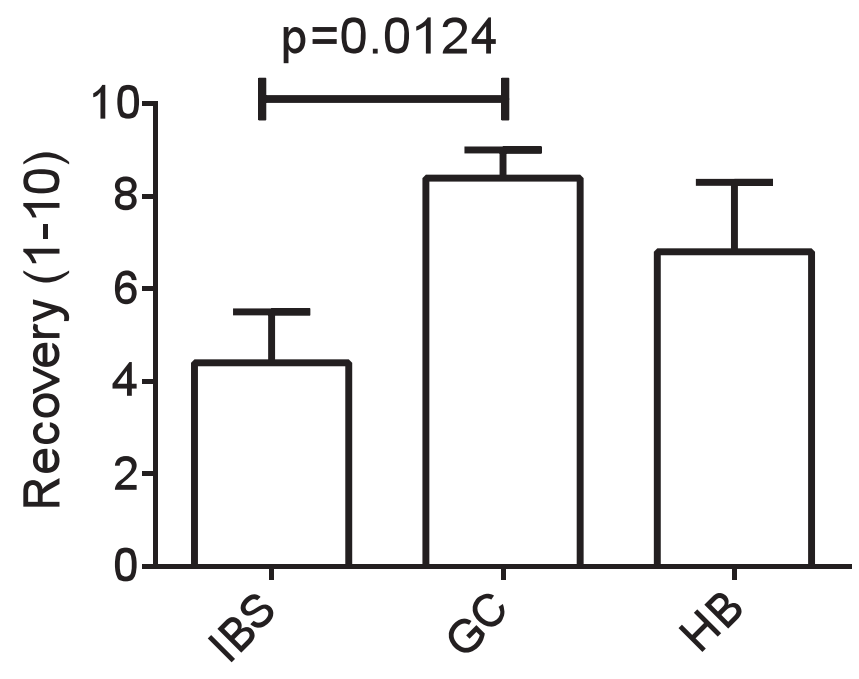

Figure 1. Recovery from IBS, GC, and HB. Statistical difference was tested by a two-tailed $t$-test.

more microbes than today, both to the total number and number of species. After finding that certain microbes were linked to serious diseases, an indiscriminate chase was started, using antibiotics, desinfectants, food preservatives, and so on. However, the underlying concept that "germ-free" humans should stay healthy showed to be completely wrong. We have instead been confronted with antibiotic resistance and depletion of the complex gut microflora.

Probiotics exert antimicrobial effects against hostile microbes by release of antimicrobial molecules and by taking up space $[1,9]$. The important benefits of probiotics come from their ability to metabolize complex carbohydrates and produce lactic acid and short chain fatty acids, such as butyric acid. Butyric acid reduces bacterial translocation, improves the organization of tight junctions, and stimulates the synthesis of mucin, a glycoprotein maintaining the integrity of the intestinal epithelium. Although no scientific studies have been conducted on FBSH in this particular perspective and probably not in any other perspectives either, interestingly, lactic acid and butyric acid are essential ingredients of FBSH.

The Helicobacter pylori bacterium is present in individuals with chronic gastritis and gastric ulcers. This bacterium is also linked to the development of duodenal ulcers and stomach cancer. $H$. pylori is present in about $50 \%$ of the world population but only causes problems in $10 \%-15 \%$. It is spiral-shaped with polar flagella that live near the surface of the human gastric mucosa. It has evolved intricate mechanisms to avoid the bactericidal acid in the gastric lumen [12]. This interaction sometimes results in severe gastric pathology. H. pylori infection is the strongest known risk factor for the development of gastroduodenal ulcers, with infection being present in $60 \%-80 \%$ of gastric and $95 \%$ of duodenal ulcers. Many researchers, including Sheu et al. [13], have demonstrated that products containing certain Lactobacillus species can reduce $H$. pylori densities in humans. Antimicrobial actions of FBSH against $H$. pylori, in particular those related to butyric acid and other short chain fatty acids, may play an important role in its efficacy against GC and HB. Interestingly, Wang et al. [14] noted that there was a clear segregation between the microbiota of colorectal cancer patients and healthy volunteers, with respect to a decrease in the abundance of butyric acid producers.

IBS is one of the most common gastroenterological diagnoses, experienced by around 11\% of the population [5]. Symptoms consist of abdominal pain associated with erratic bowel habit and variable changes in stool form and frequency, suggesting considerable heterogeneity in underlying mechanisms. Despite IBS' high prevalence, these mechanisms are poorly understood and treatment is unsatisfactory. This may explain the apparently much lower efficacy of FBSH in volunteers with IBS in comparison to those with GC or HB.

\section{Limitations in study design}

The open design of the study does not take into account the placebo effects that could be rather pronounced in a study like the present. The lack of information whether the gut symptoms have been confirmed by a physician is of course another limitation.

\section{Conclusion}

The results of the present study, in particularly those related to GC and HB, are exciting and merits further studies in volunteers, ideally in a double-blind placebo-controlled manner. In addition characterization of the active FBSH ingredients and their mechanisms of action are needed.

\section{Conflict of Interest}

The authors declare no conflict of interest.

\section{References}

[1] Patel R, DuPont HL. New approaches for bacteriotherapy: prebiotics, new-generation probiotics, and synbiotics. Clin Infect Dis 2015; 60(Suppl 2):S108-21.

[2] Duffy LC, Raiten DJ, Hubbard VS, Starke-Reed P. Progress and challenges in developing metabolic 
footprints from diet in human gut microbial cometabolism. J Nutr 2015; 145:1123S-30S.

[3] Boethius A. Something rotten in Scandinavia: the world earliest evidence of fermentation. J Archaeol Sci 2016; 66:169-80.

[4] Skåra T, Axelsson L, Stefánsson G, Ekstrand B, Hagene H. Fermented and ripened fish products in the northern European countries. J Ethnic Foods 2015; 2:18-24.

[5] Spiller R. Irritable bowel syndrome: new insights into symptom mechanisms and advances in treatment. F1000Res 2016; 5. doi:10.12688/f1000research.7992.1. eCollection 2016

[6] Savage DC. Microbial ecology of the gastrointestinal tract. Annu Rev Microbiol 1977; 31:107-33.

[7] Kussmann M, Van Bladeren PJ. The extended nutrigenomics - understanding the Interplay between the Genomes of Food, Gut Microbes, and Human Host. Front Genet 2011; 2:21; doi:10.3389/ fgene.2011.00021. eCollection 2011.

[8] Shenderov BA, Midtvedt T. Epigenomic programing: a future way to health? Microb Ecol Health Dis
2014; 25; doi:10.3402/mehd.v25.24145. eCollection 2014.

[9] Walsh CJ, Guinane CM, O'Toole PW, Cotter PD. Beneficial modulation of the gut microbiota. FEBS Lett 2014; 588:4120-30.

[10] Guarner F, Malagelada JR. Gut flora in health and disease. Lancet 2003; 361:512-9.

[11] Mitsuoka T. Intestinal flora and aging. Nutr Rev 1992; 50:438-46.

[12] Amieva MR,El-OmarEM.Host-bacterial interactions in Helicobacter pylori infection. Gastroenterology 2008; 134:306-23.

[13] Sheu BS, Cheng HC, Kao AW, Wang ST, Yang YJ, Yang $\mathrm{HB}$, et al. Pretreatment with Lactobacillus- and Bifidobacterium-containing yogurt can improve the efficacy of quadruple therapy in eradicating residual Helicobacter pylori infection after failed triple therapy. Am J Clin Nutr 2006; 83:864-9.

[14] Wang T, Cai G, Qiu Y, Fei N, Zhang M, Pang X, et al. Structural segregation of gut microbiota between colorectal cancer patients and healthy volunteers. ISME J 2012; 6:320-9. 